\title{
Outsourcing Location Selection with SODA: A Requirements Based Decision Support Methodology and Tool
}

\author{
Tommi Kramer ${ }^{1}$ and Michael Eschweiler ${ }^{2}$ \\ 1 University of Mannheim, Business School, Mannheim, Germany \\ kramer@uni-mannheim.de \\ 2 Senacor Technologies AG, Schwaig, Germany \\ michael.eschweiler@senacor.com
}

\begin{abstract}
This paper seeks to address the decision making problem in software development outsourcing scenarios in which a project manager is in charge of deciding about which software components will be outsourced and which ones will be developed internally. Therefore we propose a methodology and tool support which leverage the classification of a project's software components by means of a graph-based model of the components' requirements and their corresponding clustering. In the course of our design oriented research approach, a prototypical implementation of the methodology has been developed and evaluated. It illustrates the practical applicability of the proposed method. We thereby contribute to the location selection problem in distributed software projects and give guidance for in-house or external software production. The theoretical contribution consists of revealing an improved processing methodology for assessing software requirements and increasing the outsourcing success of a software project. Our contribution for practice is an implemented prototype for project leads of distributed teams.
\end{abstract}

Keywords: Software Development Outsourcing, Outsourcing Decision Making, Requirements Engineering.

\section{Introduction}

Information systems (IS) outsourcing has received extensive academic attention over the last twenty years with research providing insights into why firms outsource, what they outsource, which decision process they apply, how they implement their decision, and how the outcomes are handled [7. A large stake of outsourcing in practice originates from application development. Outsourcing non-critical development activities in application development can be considered as selective sourcing of application systems. Some software components are developed by internally located software teams and others are delivered by third parties from near-shore or far-shore development centers.

However, the information technology (IT) artifact as research object has experienced little academic attention in the context of outsourcing decisions. Most

C. Salinesi, M.C. Norrie, and O. Pastor (Eds.): CAiSE 2013, LNCS 7908, pp. 530-545, 2013.

(C) Springer-Verlag Berlin Heidelberg 2013 
research into outsourcing decision takes an organizational perspective, driven by major reference theories, such as transaction cost economics or the resourcebased view [7. While the identified outsourcing determinants are certainly valid in a software outsourcing context, they are insufficient to capture the whole story since the software outsourcing question is a multi-dimensional decision problem [7. A decision model for software outsourcing must additionally incorporate decision rationales derived from basic software engineering (SE) principles [14.

We therefore have set our focus on small and medium-sized enterprises (SME) in which internal resource deficits (skill-wise, personnel-wise, or performancewise) outweigh other factors as some sort of overriding contingency when taking a decision whether to outsource or not $[$. This is substantiated by a more recent study among German SME 13. This study reveals that SME mainly outsource software development striving for increased flexibility and better skill access rather than mere cost savings. Mitigating these risks requires a structured approach in the shape of a risk management process [25], for example, or simply guiding principles for a sourcing decision that enable systemic thinking [6]. This is particularly challenging for smaller firms who are characterized by less structural formalism [4. Their decisions typically evolve in some sort of collaborative ad-hoc manner. Moreover, their skills are likely to be insufficient for establishing outsourcing relationships. Also, depending on the degree and the object to be outsourced, SME might need external support that can contribute the required outsourcing expertise [2]. However, a learning curve effect can presumably be expected provided that SME frequently engage in outsourcing.

In order to address the challenges in research and practice, we have developed a decision support methodology and a tool that provide a complementary view on the outsourcing decision through the introduction of a SE perspective. Our research objects are software requirements. Our method supports the process of structuring requirements into modular clusters and deciding which of these clusters qualify for being outsourced. It builds upon a graph representation of requirements and applies spectral clustering along with graph algorithms known from social network analysis. The method is meant to provide SME with outsourcing decision support by structuring the decision problem and allowing decision makers structural analyses to classify work packages for insourcing and outsourcing. The applicability of the methodology and, hence, of the tool shall be ensured if the following requirements can be fulfilled:

1. Good clustering quality: The clusters generated by our method have to be feasible from a SE perspective (cf. clustering and cohesion).

2. Good scalability / Low setup costs: The developed methodology must scale in a larger setting with several dozens or even hundreds of requirements.

3. Perceived enhancement: The developed methodology must generate perceived utility for a decision maker in terms of enhancing his or her decision making process. This is achieved by (1) and (2) as well as further design elements (e.g. tool usability). 
Our work follows the design science research methodology [20]. In the subsequent section we step into relevant theories, concepts and existing approaches our method draws from. Afterwards, in Section 3 we describe our decision support method and its prototypical instantiation. By means of the prototype, the decision support methodology is evaluated in Section 4 before we conclude with an overall summary in the last section.

\section{Foundations and Related Work}

Our method draws from mainly three research streams that comprise information systems outsourcing, requirements engineering, and graph theory. We elaborate on the different streams in the following subsections and highlight the relevant aspects that have impact on our proposed research.

\subsection{Reference Theories in IS Outsourcing Decision Making}

The software outsourcing question which is in our focus is a multi-dimensional decision problem [7]. The majority of studies have applied transaction cost economics (TCE) to explain outsourcing behavior through conditions of market efficiency [7. However, internal resources are increasingly considered as a decisive factor as resources are heterogeneously distributed among firms and might constitute a competitive advantage, i.e. the resource-based view (RBV) adds a strategic perspective [7]. As an extension of the RBV, the knowledge-based view (KBV) stresses the importance of knowledge as differentiating resource, particularly in knowledge-intensive industries. Hence, we base our research on the proposed theoretical foundations and consider a set of derived implications in our method and tool.

Since TCE imply that the outsourcing firm has to carefully assess the specificity of any outsourcing candidate, we conclude that splitting software components with interdependent tasks is related with high communication effort and has therefore to be reduced to a minimum. Furthermore, the implications of $\mathrm{RBV}$ and KBV reveal that using external development locations for the development of organization-specific knowledge is inefficient and that outsourcing of components with high competitive relevance for a company's position on the market is inappropriate.

\subsection{Requirements Engineering}

Classification of Requirements. The prevailing distinction of requirements is into functional and non-functional requirements (NFRs). Functional requirements comprise statements on services the system must be capable to perform. Apart from the services a system offers, functional requirements define reactions to specific events or system behavior in certain situations. NFRs, on the other hand, describe constraints that include time constraints, process constraints, or standards, for example. They usually relate to the system as a whole rather 
than individual functional requirements or services. Put differently, a functional requirement describes "what" the software does in contrast to an NFR that describes "how good" a software does something.

Requirements which do not immediately pertain to the system are irrelevant for our method as its inherent model aims to semantically capture the software product, not its context. However, even system-related NFRs have to be treated carefully. NFRs constrain the way required functionality may be realized. In other words, functional requirements open up the solution space of a software system, NFRs confine it. This is due to the interdependencies that exist between requirements. While interdependencies between functional requirements rarely lead to conflicts, interdependencies between NFRs often induce unsolvable inconsistencies 22. High security and high performance are typical examples for NFRs that stand in a conflicting relationship.

A more recent distinction of requirements is represented by the classification into aspectual and non-aspectual requirements that has been brought forward through the discipline of aspect-oriented requirement engineering (AORE). AORE advances the idea of aspect-oriented programming by forming a holistic approach that embraces not only the implementation phase but also early SE phases [18. Aspect-orientation is concerned with aspects. Aspects are crosscutting concerns which exist throughout the SE process. At requirements-level, "an aspect [...] is a broadly scoped property, represented by a single requirement or a coherent set of requirements [...]" [18, p. 388]. At the stage of requirements engineering (RE), these cross-cutting concerns are referred to as early aspects because they are not necessarily transformed into what deserves the label "aspect" from a technological viewpoint, i.e. they are candidate aspects. The benefit of early aspects is to deepen the understanding of the problem domain and its semantic interdependencies [18. Aspects establish intercomponent dependencies as their cross-cutting nature makes it necessary that components provide services to or require services from other components. Typical examples for functional aspects are capabilities which are required throughout a software system, such as persistency, collaboration, synchronization or locking mechanisms.

Requirements Interdependencies. By focusing on requirements, we argue that it is crucial for software clustering and architectural design to understand a system from a semantic perspective, independent of syntactic dependencies. Hence, our method attempts to capture relevant types of semantic interdependencies that exist between requirements.

Generally, such interdependencies can be classified into vertical and horizontal ones as illustrated by Figure 1. Vertical dependencies stem from transitions between RE phases that require a change of the level of abstraction. These vertical relations are mostly hierarchical in that lower level requirements refine what a higher level requirement specifies. A simple example for a refinement is a feature edit that is decomposed into copy, paste, and delete. Because these relationships represent transitions between different degrees of abstraction of the same requirement, they are not of interest for the method we present in this paper. Instead, the focus is on horizontal dependencies that constitute system cohesion 
SL 0

SL 1

SL 2

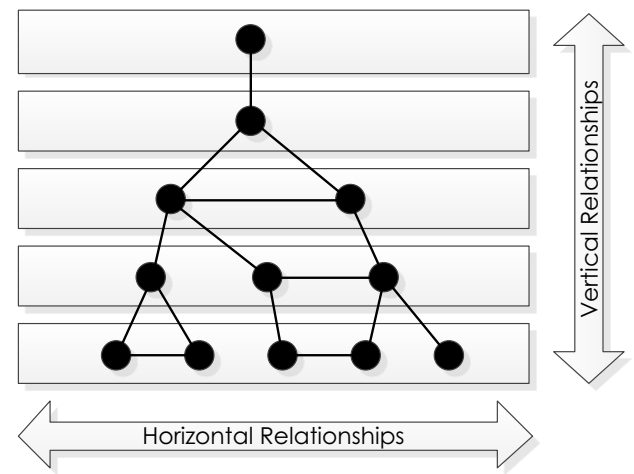

SL Specification Level

- Requirement

- Relationship

SL 4

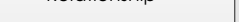

Fig. 1. Specification levels and relationships between requirements (based on 22])

and impact on how requirements are semantically related and how they can be structured, correspondingly.

We refer to a model of seven fundamental interdependency types [5] that synthesizes different views from the literature. In that definition, similar_to refers to a semantic match of varying degree and is significant for structuring requirements as it is an expression of cohesion between two requirements. Requires describes the condition that "[the] fulfillment of one requirement depends on the fulfillment of another requirement" [5, p. 103]. Beside conditional and functional dependencies, requires also describes temporal dependencies in the form that a requirement needs to be implemented before another one can be implemented. In summary, requires is highly relevant as it alters the semantics of interlinked requirements and thus impacts on their cohesion with varying intensity. Our method focuses on the relations requires and similar_to which are considered the main drivers of semantic cohesion.

\subsection{Graph Theory}

Graph Representation. Graph theory essentially relies on the existence of pairwise relations between objects. Requirements and their interdependencies exactly represent such objects with pairwise relations in between. Hence, graph theory is ideally suited for the formal representation of requirements and their relations. The formal graph representation provides the necessary degree of structure for the computable part of the decision problem, the clustering of requirements and corresponding metrics. Hence, we represent requirements by the use of typed and weighted graphs where types describe different sorts of interdependencies and weights express varying degrees of cohesion. To work with these graphs programmatically, we use a weighted adjacency matrix.

Graph Partitioning. Clustering vertices into disjoint subsets is known as graph partitioning in graph theory. The properties quality and time of graph 
partitioning heuristics were found to stand in a conflicting relationship. By tendency, algorithms that take longer produce better results than more time-efficient algorithms. Whereas this is a trade-off in time-critical settings, such as parallel computing, time is not that much of an issue in the context of our decision problem. As a logical consequence, high partitioning quality is the primary intent. Partitioning quality, in turn, can be defined in various ways. For graph partitioning in general, it is usually determined by the cut size. Given two disjoint partitions V1 and V2, the cut describes all edges that have one end in V1 and the other in V2. Its size is the sum of edges or, given they are weighted, the sum of their weights. Typically, partitioning algorithms try to minimize the cut size.

Graph-partitioning algorithms can be classified into geometric and coordinatefree algorithms [10. In the context of graphs, geometric algorithms require a graph to be embedded into the metric space which is not given in the present case. Requirements do not possess any coordinates. This fact rules out geometric algorithms leaving non-geometric heuristics. These coordinate-free methods focus on the combinatorial structure of the graph [10] which, from the perspective of this paper, replicates the semantic cohesion of requirements. We focus on the set of algorithms from the field of recursive spectral bisection (RSB). By tendency, RSB algorithms outperform traditional approaches, are easy to implement, and efficient to solve through standard linear algebra operations [17]. They leverage the algebraic properties of a graph's matrix representation, particularly that of its Laplacian matrix.

Structural Analysis of Graphs. Structural analysis of graphs uses metrics to derive conclusions about the characteristics of the whole graph, subgraphs, or individual vertices. Whereas algorithms process the graph structure in order to solve a certain problem, structural analysis generates quantitative measures in order to describe it and the construct it has been derived from. This is particularly useful for the given problem as it allows to objectively identify important and less important requirements on the basis of their relations and weights. To do so, we make use of the centrality concept that originally stems from the analysis of communication networks and tries to identify nodes that are important to the communication within the network.

\subsection{Related Work}

Holistic research into the question of what parts of a software system, that is yet to be developed, qualify for outsourcing is scarce. Hence, our review of related work follows our method's three-step approach: graph-based representation of requirements, clustering of requirements and structural analysis of requirements.

Graph-Based Representation of Requirements. Approaches towards the graph-based representation of requirements mainly include graph-based traceability and the representation of NFRs. The latter mostly deals with decision making but is not in our focus. The field of graph-based traceability attempts to leverage the benefits of a graph-based visualization for improving the ability to 
explore and analyze requirements and their interrelationships, e.g., a graph-based model that uses labeled edges to represent requirements. The model contains a weight measure for edges that express the semantic match between different requirements 1112. Schwarz et al. 23. present a more recent and comprehensive approach to apply a formal graph representation in order to improve traceability. They used typed edges to distinguish between different sorts of interdependencies. Li et al. 15] presented a graph-based approach for change impact analysis as part of requirements traceability. They used different types of associations that are based on an older version of the model of fundamental interdependency types [5]. Only the approach by Yaung [26] could be identified as using a graph-based model for the purpose of requirements clustering. His approach also focuses on functional requirements without distinguishing between relationship types. A relation in his model expresses a certain degree of cohesion. The degree is included as an edge weight.

Requirements Clustering. The majority of requirements clustering approaches use clustering for the purpose of system modularization which is commonly viewed as decomposition into strongly cohesive and loosely coupled groups of requirements. However, the individual approaches to arrive at this state differ substantially. Li et al. [16] aimed at requirements encapsulation which, in essence, is the modularization of requirements and the definition of interfaces for these modules. They defined a set of seven requirements attributes that pertain to semantics and structure. They did not use explicit relations between requirements.

Requirements are clustered based on the overall similarity of their attributes, hence multi-dimensional similarity. Their approach requires a detailed requirements specification and extensive manual work in order to define the set of requirements and their attributes. The approach of Yaung [26] is presumably closest to our objective of clustering a graph structure into cohesive groups of nodes. His approach is, however, simpler in that he did not distinguish between different types of relations. Furthermore, the algorithm he proposed requires the specification of a cohesion threshold by an expert. If the cohesion of two requirements is above that threshold, they are assigned into the same cluster. Hence, the result of the algorithm is highly dependent on how that parameter has been defined. Nonetheless, it is an early example of the applicability of a graph-based approach.

Finally, some additional attempts to cluster a requirements similarity matrix for the purpose of software modularization have been identified [1]. It is, however, difficult to draw a conclusion on how the algorithms competitively perform.

Structural Analysis of Requirements. Structural metrics in the context of graphs give insights into the characteristics of a graph (global measures) or certain vertices (point/local measures). Of interest are centrality measures that reflect the importance of a vertex in a network. The analysis of such networks has become popular in social sciences as social network analysis (SNA). Within graph-based traceability, social network analysis (SNA) has been applied but with emphasis on important persons within the traceability network [12]. 
Only one approach could be identified that applies SNA techniques to assess the importance of individual requirements. Fitsilis et al. 9] attempted to conduct a prioritization through structural metrics. To do so, they created a requirements interdependency matrix which was then used to determine the individual centrality measures. They demonstrated the results for betweenness centrality, closeness centrality, and different types of degree centrality (in, out, total). Results indicated that different centrality metrics yield different values, i.e. rankings varied to a certain degree depending on the centrality measure chosen. While their example illustrates the applicability of this approach, it also demonstrates that this field is understudied. It requires standard measures along with reference values to describe the characteristics of requirements throughout the SE process 9.

\section{SODA - A Decision Support Method}

\subsection{Method Overview}

Figure 2 sketches the conceptual steps that constitute the SODA (Software Outsourcing Decision Aid) method. The first step relates to the creation of the requirements model. Software requirements are transformed into a graph-based model which is based on specific syntactic rules. The graph-based model is then forwarded to the second step that attempts to identify cohesive groups of requirements through the application of a clustering algorithm. The third step takes the clusters that have been found and conducts a structural analysis in order to determine each cluster's outsourcing suitability.

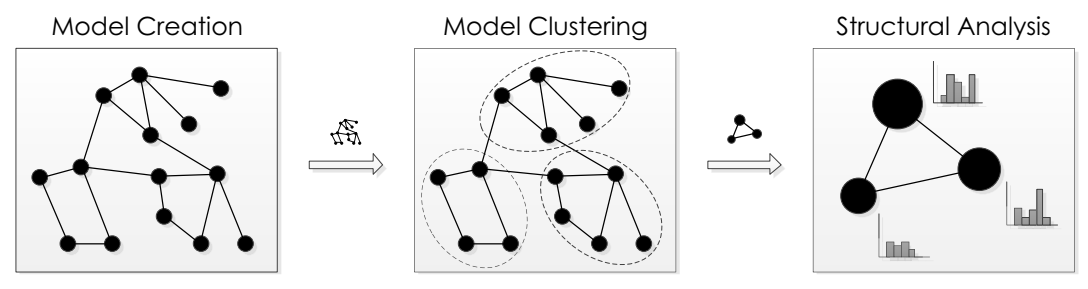

Fig. 2. Overview of the SODA method

\subsection{Representing Requirements}

The requirements model (RM) is meant to reflect the combinatorial structure of a software project's requirements. Only horizontal relations ought to be included. Dahlstedt's and Persson's model of fundamental interdependency types [5] has been narrowed down to the interdependency types requires and similar_to. Our graph-based requirements model is defined as

$$
G_{R M}=\left(V, E, w_{E}, t_{E}, W, T\right)
$$


where $V$ represents the set of requirements, $E$ represents the multiset of directed edges that refer to the interdependencies between the requirements in $V . w_{E}$ is a weight function that assigns a weight to each edge in $E$ reflecting an interdependency's strength. We suggest three degrees of dependency strengths. How these three degrees are transformed into quantitative values ultimately depends on the specific implementation of SODA. The only restriction imposed here postulates that $W$ may not contain negative elements. $G_{R M}$ is a labeled graph. Hence, $t_{E}$ is a map that assigns a type, i.e. label, to each edge in $E$ from the set of types $T . T$ is defined as $T=\{$ similar_to, requires $\}$. It can be easily extended by simply adding additional interdependency types to $T$. Through this strategy, new perspectives can be included into the model. This allows clustering the model based on selected interdependency types to investigate differences between decompositions under selected decomposition criteria. Put differently, the model can be considered as a layered model with each layer being spanned by a specific interdependency type. The general model aggregates all types in a single graph. A typed model thus represents an excerpt of the general model. The model does not allow loops. For the given set of interdependency types, loops generate no informative value. Figure 3 gives an impression of how the requirements model is presented in our prototype. The size of each requirement is derived from its centrality.

An important aspect that shall be briefly mentioned pertains to the population of our requirements model. From a cost perspective, SODA's main cost driver is the identification of interdependencies between requirements. Unless (semi)automatic approaches are at hand to support the identification activity, pairwise comparisons must be conducted at the cost of $(n *(n-1)) / 2$ where $n$ is the number of requirements.

\subsection{Structuring Requirements}

The objective of the clustering step is to find cohesive groups of requirements. In the case of software outsourcing neither the number of clusters nor a cluster's size is known a priori. A major aspect is that semantically cohesive groups of requirements are not necessarily equally sized. Also, the number of clusters is to be unconstrained. Setting it would improperly bias the algorithm and prevent it from finding a partition that is optimal from SODA's objective viewpoint. Hence, in the present case, the algorithm needs to be non-parameterized and free of externally imposed constraints. Its sole input represents the structure that is to be divided.

We selected an algorithm developed by Newman [19. It is particularly applicable for the research problem as it is non-parameterized and attempts to maximize the modularity of a given network. The objective of Newman's algorithm [19] can be referred to as community structure detection. A community structure denotes the appearance of "[...] densely connected groups of vertices, with only sparser connections between groups" [19, p. 8577]. An algorithm that detects community structures respects that the number and size of communities are determined by the network rather than a supervisor. It also admits the fact that 


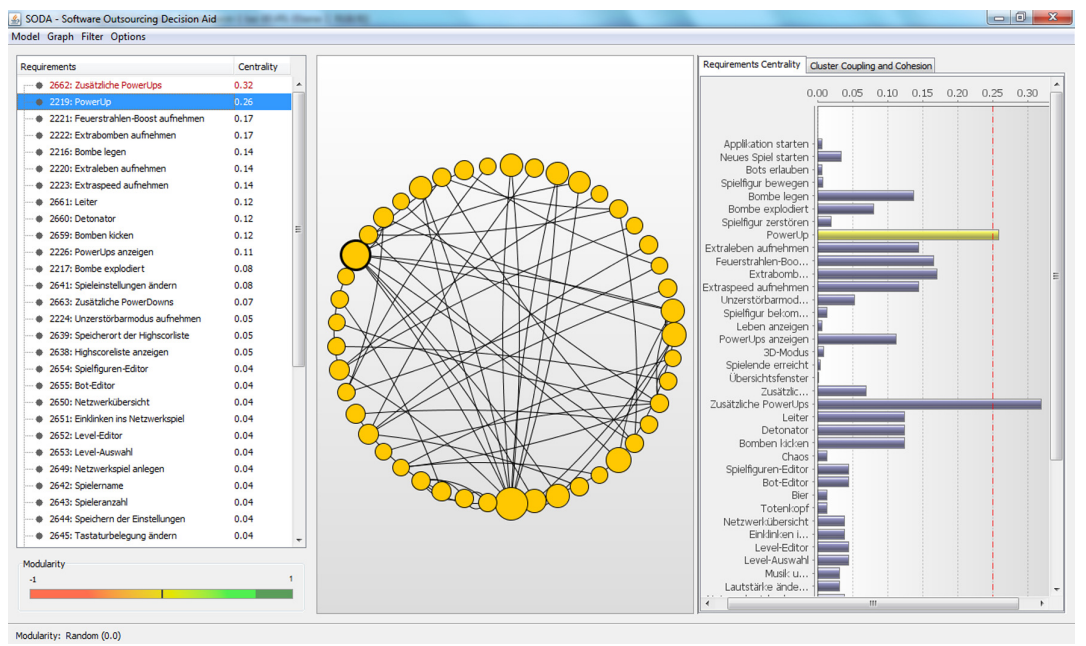

Fig. 3. SODA prototype before requirements clustering

there might not be any suitable division of the network. This criterion is decisive for SODA and could not be identified in any of the other spectral algorithms as they usually force partitions into a prescribed size tolerance. Newman [19], however, loosened this constraint through a redefinition of the Laplacian matrix. Another advantage is that his algorithm automatically determines the number of clusters through the inclusion of a control measure: modularity. Newman's approach [19] is thus tailored to the objective of identifying modular communities which are unbalanced in terms of size. We combine his global partitioning algorithm with a variant of the Kernighan-Lin algorithm he proposes as well. It conducts a local optimization of the modularity measure through movements of individual vertices between pairs of clusters. Figure 4 shows our prototype after clustering the set of requirements.

\subsection{Structural Analysis of Requirements}

Global: Modularity. The objective of SODA's third step is to take the requirements structure that has been algorithmically determined in step two and analyze it from a global (whole graph), regional (cluster), and local perspective (vertex) in order to guide a human decision maker in his or her outsourcing decision. A global metric can be derived from Newman's clustering algorithm [19] in order to characterize the modularity $Q_{P}$ of a given network partition $P$. This allows putting it into relation to other partitions of the same network or reference values that might stem from other projects, for example. Modularity is quantitatively assessed through a pairwise comparison of nodes. If two nodes fall within the same group, their contribution to modularity is the weight of the edges between them minus the expected weight in an equivalent network with randomly placed edges [19]. 
Through this approach, global modularity is dependent on the partition of the network. If a user decides to manually change the partition that has been proposed by the algorithm, he or she can observe the corresponding impact on modularity. Altering the partition proposed by the algorithm cannot improve modularity as measured here since the algorithm determines the partition that maximizes $Q$. Hence, $Q_{\max }$ shall denote the achievable modularity for a specific requirements model. It is computed using the partition generated by SODA's clustering algorithm. Any manual movement of nodes impairs $Q_{P}$. Nonetheless, there might be valid reasons to alter a generated partition as an individual might base his or her decision on additional information which is not included in the model.

The value range of $Q_{P}$ stretches from -1 to +1 . $Q_{P}$ ranging around zero indicates a random distribution of edges with no identifiable community structure. For networks that exhibit a perceivable community structure, $Q_{P}$ typically ranges between 0.3 and 0.7 . Networks with negative $Q_{P}$ have weakly cohesive groups that have fewer intra-linkages than one would expect in a random graph. As indicated, $Q_{\max }$ sets an upper bound on the scale from -1 to +1 for a given requirements model. It is a first indicator of how interdependent a project's requirements are from a global viewpoint. Regional and point metrics then allow creating a more differentiated picture of the situation.

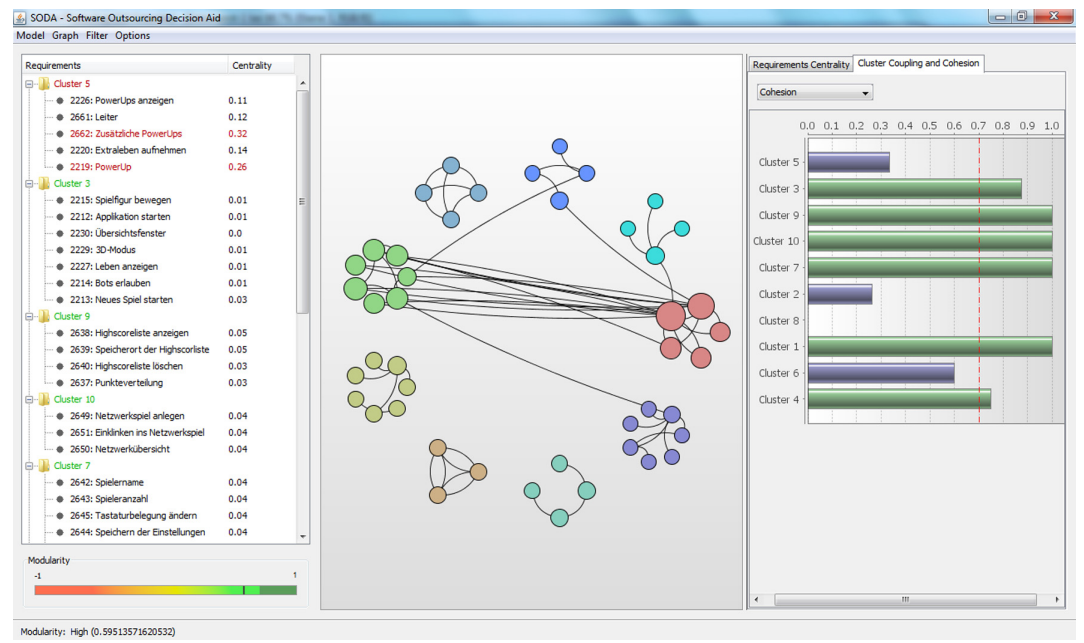

Fig. 4. SODA prototype after requirements clustering

Regional: Cluster Coupling and Cohesion. Cluster coupling and cohesion represent regional or group metrics. The former is an indicator of how strongly related the responsibilities of a subset of requirements are. The latter expresses how strongly a subset is connected to or requires external requirements. Whereas $Q_{\max }$ characterizes the entire model, coupling and cohesion describe groups of 
requirements, specifically the clusters that have been generated in SODA's partitioning algorithm. Coupling and cohesion hence provide a deeper insight into the outsourcing suitability of each cluster. Briand et al. 3. formulated properties for functions that measure coupling and cohesion to which our proposed metrics adhere.

For the expression of a cluster's degree of coupling, we propose a simple countbased measure that cumulates the weight of all inter-linkages of that cluster to other clusters. It is deliberately not a normalized measure and thus has a value range from 0 to positive infinity. The underlying notion is that coupling is independent of both the size of the cluster and the project and only determined by the weight of linkages to other clusters.

Unlike coupling, cohesion is not an additive metric. Instead, Briand et al. 3 stress that cohesion is a normalized metric that is forced into a specific interval. Hence, we propose a function that puts the weight of all intra-linkages into relation to the weight of all intra- and inter-linkages of a specific cluster. The value range is thus constrained to the interval from 0 to 1 . Cohesion of below 0.5 indicates that a cluster of requirements has more external than internal interdependencies. Hence, it is desirable to have a cohesion that lies significantly over 0.5 and approaches 1 . It is unlikely to achieve maximum cohesion for all clusters due to the near decomposability property of complex systems [24].

Local: Requirements Centrality. For SODA, the importance of a requirement is essentially dependent on its position in the combinatorial structure of the model. This position can be characterized by the weight of its interdependencies to other requirements as well as the importance of these neighboring requirements. In that sense, importance is reciprocal. Centrality is thus driven by differences in degree. The group of centrality measures that reflects these aspects is feedback centrality. We have adopted the eigenvector centrality in which larger components of a graph are by tendency weighted more strongly than smaller ones. As previously stressed, there is a lack of reference values regarding the application of network analysis measures for requirements analysis. Hence, SODA does not define a threshold that allows a clear statement whether a specific requirement might critical, i.e. too important to be outsourced.

Rule-Based Recommendations. SODA does not aim at deciding which requirements can be outsourced and which cannot. Its role is meant to be supportive in the sense that decision makers get an understanding of the project's semantic structure, relevant interdependencies, and the role of individual requirements. In the end, it is up to the decision maker to derive a final conclusion his or her decision can be grounded on. As a logical conclusion, SODA is a means to explore the decision problem. Through the definition of threshold values for eigenvector centrality, coupling, and cohesion it allows for a rule-based identification of requirements clusters that represent outsourcing candidates. The quality of these identified outsourcing candidates ultimately depends on the ability of the decision maker to set these thresholds and judge the situation. It is to a large extent subject to his or her experience. 


\section{Evaluation and Discussion}

The method which is subsequently applied is a mixture of experimental and descriptive approaches. The experimental simulation addresses the formal core of our decision support method and provides evidence for fulfilling the solution criteria (clustering quality, scalability, and enhancement) as introduced in Section 1. The simulation was performed using data from four student projects. The projects have been conducted within the context of a lecture for master students in information systems. Students were meant to develop a computer game based on prescribed requirements. The project teams had to use a collaboration platform and were incentivized to exploit its functionality, so all of them maintained requirements interdependencies. These were recorded based on the students' judgment of what are adequate semantic relations. This data set was particularly suitable to investigate how a varying combinatorial structure of semantically identical sets of requirements determined the prototype's output.

Hence, our focus was on any observable correlations between the number of interdependencies and the method's output figures. We could observe that more interdependencies led to a more coarse-grained partition of the graph. Achievable modularity $\left(Q_{\max }\right)$ turned out lower as a logical result of the algorithm finding only larger and hence fewer community structures. The question that came up here was how clustering quality is affected by that constellation. To investigate this, we conducted another test and applied the Rand index to measure the quality of the clustering algorithm [21. The results are shown in Table 1 below. The ground truth was defined based on our perception of what would be a sensible partition.

Table 1. Rand index for measurement of clustering quality

\begin{tabular}{l|l|l|l|l|l}
\hline Project & $\begin{array}{l}\text { Require- } \\
\text { ments }\end{array}$ & $\begin{array}{l}\text { Interdepen- } \\
\text { dencies }\end{array}$ & $\begin{array}{l}\text { Achievable } \\
\text { Modularity }\end{array}$ & $\begin{array}{l}\text { No. of Clusters in } \\
\text { Optimal Partition }\end{array}$ & $\begin{array}{l}\text { Rand } \\
\text { index }\end{array}$ \\
\hline $\mathrm{A}$ & 45 & 61 & 0.71 & 10 & 0.80 \\
$\mathrm{~B}$ & 45 & 43 & 0.67 & 8 & 0.84 \\
$\mathrm{C}$ & 45 & 181 & 0.54 & 6 & 0.77 \\
$\mathrm{D}$ & 46 & 49 & 0.65 & 8 & 0.82 \\
\hline
\end{tabular}

The differences in the individually generated partitions that can be observed mainly consist in more fine-grained partitions than this is the case for the ground truth. Simply merging individual clusters gives a good approximation of the ground truth which is why the Rand index reports a good clustering quality for all projects. Project $\mathrm{C}$ which has by far the most interdependencies also exhibits the coarsest granularity with only six clusters in its partition. Whether this is desirable is speculative. What can be said is that group C put the most effort 
into interdependency maintenance. The benefit of this investment is not clear. Judged from the viewpoint of scalability, this is encouraging with respect to the effort necessary to set up the prototype. It suggests that even in a business environment, the required effort might lie within manageable boundaries because fewer interdependencies apparently allow for results of satisfying quality. In contrast, project D featured 46 requirements with significantly less interdependencies than $\mathrm{C}$. Beside the surprisingly good clustering quality as indicated by the Rand index, an important finding is that clustering quality is not necessarily better in a model that has a larger set of interdependencies. This is particularly promising given the previously mentioned problem of costly interdependency identification. Thus, we can infer from our experimental simulation that the postulated requirements of good clustering quality and scalability (cf. Section 1) are being fulfilled by our method under laboratory conditions.

The third requirement of perceived enhancement could not be validated in these laboratory conditions. We believe that SODA's major weaknesses are located in the last step that pertains to the structural analysis of requirements clusters and the subsequent recommendation of outsourcing candidates. Although the rule-based approach assures consistency, its expressiveness is limited due to the fact of scarce research. Moreover, SODA is highly dependent on human input as the quality of its suggestions is essentially induced by the quality of the underlying model and the threshold values that have been defined by the user. With regard to the model, we can conclude that the inferencing capabilities are driven by the quality of the underlying interdependencies. Interdependencies are the most critical element within the decision support method developed here. They predominantly determine the combinatorial structure of the model. Hence, the results SODA produces are very sensitive to the input it receives. Another problematic aspect is the lack of reference values for outsourcing decisions. As a logical conclusion, the quality of SODA's recommendations is similarly sensitive to the threshold values as it is to the model itself.

It shall be stressed that SODA does not propose a software architecture. It is grounded on the argumentation that it is beneficial to understand a software system from a semantic perspective. Clustering of requirements can lead to a modular design [16] but design is subject to more factors than just semantic relations. To investigate its potential outside of the laboratory, we plan to conduct a qualitative case study that builds upon requirements data from a business setting and benefits from expert judgments, especially targeting at evaluating to what extent SODA does perceivably enhance the outsourcing decision process in a SME.

\section{Conclusion}

We have developed SODA, a decision support method and tool that support decision makers in analyzing the outsourcing suitability of requirements from a SE perspective and leverage the location dependent sourcing decision problem. SODA builds on existing research in that it represents a cross-discipline 
artifact that draws from the research streams of information systems outsourcing, requirements engineering, and graph theory. SODA constitutes a three-step approach, including graph-based modeling of requirements and their semantic interdependencies, model clustering, and structural analysis of clusters and requirements through a set of structural metrics. Based on these metrics, it performs a rule-based recommendation of outsourcing candidates.

The evaluation result reflects the novelty, robustness and scalability of the approach. Overall, it indicates that SODA is applicable for the identification of outsourcing candidates. This, however, needs to be interpreted in a differentiated manner. Its strengths are located in the representation and clustering of requirements. Its main weakness pertains to the set of structural metrics. Because this field is notoriously understudied, there is little knowledge SODA can draw from which induces a substantial degree of uncertainty. Furthermore, a qualitative case study will shed more light on the practical usefulness of our approach.

\section{References}

1. Al-Otaiby, T.N., AlSharif, M.: Software requirements modularization using partitioning clustering technique. In: Proceedings of the 45th Annual Southeast Regional Conference, pp. 65-69 (2007)

2. Al-Qirim, N.A.Y.: The strategic outsourcing decision of it and ecommerce: The case of small businesses in new zealand. Journal of Information Technology Cases and Applications 5(3), 32-56 (2003)

3. Briand, L., Morasca, S., Basili, V.: Property-based software engineering measurement. IEEE Transactions on Software Engineering 22(1), 68-86 (1996)

4. Carmel, E., Nicholson, B.: Small firms and offshore software outsourcing: High transaction costs and their mitigation. Journal of Global Information Management 13(3), 33-54 (2005)

5. Dahlstedt, S.G., Persson, A.: Requirements interdependencies: State of the art and future challenges. In: Aurum, A., Wohlin, C. (eds.) Engineering and Managing Software Requirements, pp. 95-116 (2005)

6. Dedrick, J., Carmel, E., Kraemer, K.L.: A dynamic model of offshore software development. Journal of Information Technology 26, 1-15 (2011)

7. Dibbern, J., Goles, T., Hirschheim, R., Jayatilaka, B.: Information systems outsourcing A survey and analysis of the literature. Communications of the ACM 35(4), 6-102 (2004)

8. Dibbern, J., Heinzl, A., Leibbrandt, S.: Interpretation des Sourcings der Informationsverarbeitung: Hintergründe und Grenzen ökonomischer Einussgrößen. Wirtschaftsinformatik 45(5), 533-540 (2003)

9. Fitsilis, P., Gerogiannis, V., Anthopoulos, L., Savvas, I.: Supporting the requirements prioritization process using social network analysis techniques. In: 19th IEEE International Workshop on Enabling Technologies: Infrastructures for Collaborative Enterprises (WETICE), pp. 110-115 (2010)

10. Fjällström, P.O.: Algorithms for graph partitioning: A survey. Linköping Electronic Articles in Computer and Information Science 3(10), 1-37 (1998)

11. Heim, P., Lohmann, S., Lauenroth, K., Ziegler, J.: Graph-based visualization of requirements relationships. In: Requirements Engineering Visualization, pp. 51-55 (2008) 
12. Hildenbrand, T.: Improving Traceability in Distributed Collaborative Software Development. Lang, Frankfurt (2008)

13. Klimpke, L., Kramer, T., Betz, S., Nordheimer, K.: Globally distributed software development in small and medium-sized enterprises in germany: Reasons, locations, and obstacles. In: Proceedings of the 19th European Conference on Information Systems (ECIS 2011), Helsinki, Finland (2011)

14. Kramer, T., Heinzl, A., Spohrer, K.: Should this software component be developed inside or outside our firm? - A design science perspective on the sourcing of application systems. In: Kotlarsky, J., Willcocks, L.P., Oshri, I. (eds.) Global Sourcing 2011. LNBIP, vol. 91, pp. 115-132. Springer, Heidelberg (2011)

15. Li, Y., Li, J., Yang, Y., Li, M.: Requirement-centric traceability for change impact analysis: A case study. In: Wang, Q., Pfahl, D., Raffo, D.M. (eds.) ICSP 2008. LNCS, vol. 5007, pp. 100-111. Springer, Heidelberg (2008)

16. Li, Z., Rahman, Q.A., Ferrari, R., Madhavji, N.H.: Does requirements clustering lead to modular design? In: Glinz, M., Heymans, P. (eds.) REFSQ 2009. LNCS, vol. 5512, pp. 233-239. Springer, Heidelberg (2009)

17. Luxburg, U.: A tutorial on spectral clustering. Statistics and Computing 17(4), 395-416 (2007)

18. Moreira, A., Araújo, J.: The need for early aspects. In: Fernandes, J.M., Lämmel, R., Visser, J., Saraiva, J. (eds.) GTTSE 2009. LNCS, vol. 6491, pp. 386-407. Springer, Heidelberg (2011)

19. Newman, M.E.J.: Modularity and community structure in networks. Proceedings of the National Academy of Sciences of the United States of America 103, 8577-8582 (2006)

20. Peffers, K., Tuunanen, T., Rothenberger, M.A., Chatterjee, S.: A design science research methodology for information systems research. Journal of Management Information Systems 24(3), 45-78 (2007)

21. Rand, W.M.: Objective criteria for the evaluation of clustering methods. Journal of the American Statistical Association 66(336), 846-850 (1971)

22. Rupp, C.: Requirements-Engineering und -Management, 4th edn. Carl Hanser Verlag, Munich (2009)

23. Schwarz, H., Ebert, J., Winter, A.: Graph-based traceability: a comprehensive approach. Software and Systems Modeling 9(4), 473-492 (2010)

24. Simon, H.A.: The architecture of complexity. Proceedings of the American Philosophical Society 106, 467-482 (1962)

25. Yalaho, A.: A conceptual model of ict-supported unified process of international outsourcing of software production. In: 10th IEEE International Enterprise Distributed Object Computing Conference Workshops, EDOCW, pp. 47-58 (2006)

26. Yaung, A.T.: Design and implementation of a requirements clustering analyzer for software system decomposition. In: Proceedings of the 1992 ACM/SIGAPP Symposium on Applied Computing: Technological Challenges of the 1990s, pp. 1048-1054 (1992) 